\title{
Shock formation in the compressible Euler equations and related systems
}

\author{
Geng Chen* Robin Young ${ }^{\dagger} \quad$ Qingtian Zhang ${ }^{\ddagger}$
}

\begin{abstract}
We prove shock formation results for the compressible Euler equations and related systems of conservation laws in one space dimension, or three dimensions with spherical symmetry. We establish an $L^{\infty}$ bound for $C^{1}$ solutions of the one-D Euler equations, and use this to improve recent shock formation results of the authors. We prove analogous shock formation results for one-D MHD with orthogonal magnetic field, and for compressible flow in a variable area duct, which has as a special case spherically symmetric three dimensional flow on the exterior of a ball.
\end{abstract}

2010 Mathematical Subject Classification: 76N15, 35L65, 35L67.

Key Words: Conservation laws, singularity formation, compressible Euler equations, MHD, large data.

\section{Introduction}

One of the defining features of systems of hyperbolic conservation laws,

$$
u_{t}+f(u)_{x}=0, \quad(x, t) \in \mathbb{R} \times \mathbb{R}^{+}, u \in \mathbb{R}^{n},
$$

in the absence of regularizing dissipative effects such as viscosity and heat loss, is the blowup of gradients and subsequent formation of shock waves.

\footnotetext{
* Department of Mathematics, Pennsylvania State University, University Park, PA 16802 (chen@math.psu.edu).

${ }^{\dagger}$ Department of Mathematics and Statistics, University of Massachusetts, Amherst, MA 01003 (young@math.umass.edu). Supported in part by NSF Applied Mathematics Grant Number DMS-0908190.

${ }^{\ddagger}$ Department of Mathematics, Pennsylvania State University, University Park, PA 16802 (zhang_q@math.psu.edu).
} 
Once a shock forms, discontinuous weak solutions must be considered, and the corresponding loss of regularity makes the systems hard to analyze.

We are concerned with the conditions under which a shock forms in a classical $C^{1}$ solution of (1.1). For pairs of equations $(n=2)$, Lax showed in [13, 14] that shocks always form for data which is anywhere compressive. In work initiated by John, this was extended to larger systems, given restrictions on the size of the initial data [11, 16, 21]. In particular, all waves in those solutions are assumed to be weak. One of our goals is to remove these severe restrictions on the initial data, which includes allowing for large waves in the solution. We restrict our attention to systems arising from physical problems because of the variety of unstable phenomena which can affect general $3 \times 3$ systems when the data is allowed to be large $[9,10,27,28,30$.

Recently, the authors have obtained large data results for the $3 \times 3 \mathrm{com}$ pressible Euler equations [2, 4]. In all of these works, (1.1) is differentiated and manipulated to get a system of PDEs with quadratic nonlinearities for the evolution of gradients along characteristics. For two equations, these PDEs decouple to yield a pair of Riccati type ODEs along characteristics; the quadratic nonlinearity then implies that the derivatives must blow up in finite time [13, 14]. For general systems, if the data has small total variation, the nonlinear waves separate in finite time, after which they are essentially decoupled and genuine nonlinearity in each field causes gradients to blow up by the same mechanism.

In [2, 4], we consider the compressible Euler equations, and make a series of nonlinear changes of variables to get an uncoupled pair of quadratic ODEs for the genuinely nonlinear fields, which exhibit the same blowup mechanism. Although there are no restrictions on the size of the initial data, the results of [2, 4] rely on assumed global bounds for the state variables. In this paper, we first remove this restriction by obtaining an a priori estimate on the state variables for $C^{1}$ solutions of the Euler equations, yielding a condition on only the initial data which guarantees blowup of gradients. See [19] for a description of attempts to obtain a stronger $L^{\infty}$ bound and global existence which were unsuccessful.

We then extend the method to larger systems of physical interest. We show similar gradient blowup results for one-dimensional magnetohydrodynamics (MHD) with orthogonal magnetic field, and compressible flow in a variable area duct. As a special case, we obtain a gradient blowup result for spherically symmetric three-dimensional Euler flow on the exterior of a ball. These blowup results again rely on global bounds for the state variables, but in view of our obtained bounds for the compressible Euler equations, we expect that this is just a technical assumption. Indeed, we 
note that all known cases of blowup of state variables occur in non-physical systems [9, 10, 27, 28, 30].

The mechanism for gradient blowup and shock formation is the steepening of compressions due to genuine nonlinearity: if the wavespeed is larger behind the wave, it will eventually break. For $2 \times 2$ systems (or diagonal systems such as chromatography), the different wave families weakly decouple and all compressions will collapse into shocks. However, in $3 \times 3$ systems, and especially for large data, the wave interactions between different families influence this process and can delay the onset of shocks [18, 20, 25]. In the context of Euler, the contacts act as partial reflectors of waves and with care one can set up solutions in which compressions are cancelled before they focus [25, 5].

Our conditions which guarantee blowup are expressed in terms of global bounds for the variables, so we must ensure that the state does not leave some compact set. In particular, for a $\gamma$-law gas, the vacuum must be avoided. Moreover, when $1<\gamma<3$, our estimates depend on a lower bound for the density as well as on the upper bound. This is also true in the isentropic case treated by Lax.

The paper is organized as follows: in Section 2, we obtain a priori $L^{\infty}$ bounds for the density and velocity in the one-D compressible Euler equations. We then use this to stregthen the gradient blowup result of [2]. In Section 3, we consider one-D MHD with orthogonal magnetic field, derive Riccati type equations for gradients, and use these to obtain a stronger singularity formation result than that of [4. In Section 4, we similarly derive Riccati type equations and present a shock formation theorem for compressible Euler flow in a variable area duct. This includes the special case of three-D spherically symmetric flow in the exterior of a ball.

\section{Compressible Euler equations}

We begin by considering the initial value problem for the compressible Euler equations in a Lagrangian frame in one space dimension,

$$
\begin{aligned}
\tau_{t}-u_{x} & =0, \\
u_{t}+p_{x} & =0, \\
\left(\frac{1}{2} u^{2}+e\right)_{t}+(u p)_{x} & =0,
\end{aligned}
$$

where $\rho$ is the density, $\tau=\rho^{-1}$ is the specific volume, $p$ is the pressure, $u$ is the velocity, $e$ is the internal energy, and $x$ is the material coordinate. The 
system is closed by the Second Law of Thermodynamics,

$$
T d S=d e+p d \tau
$$

where $S$ is the entropy and $T$ the temperature. For $C^{1}$ solutions, it follows that (2.3) is equivalent to the "entropy equation"

$$
S_{t}=0
$$

When the entropy is constant, the flow is isentropic, and (2.1) and (2.2) become a closed system, known as the $p$-system [24].

We assume the gas is ideal polytropic, so that

$$
p \tau=R T \quad \text { and } \quad e=c_{\tau} T
$$

with ideal gas constant $R$, and specific heat $c_{\tau}$; this implies

$$
p=K e^{\frac{S}{c_{\tau}}} \tau^{-\gamma}
$$

with adiabatic gas constant $\gamma=\frac{R}{c_{\tau}}+1$, see [6]. The nonlinear Lagrangian sound speed is

$$
c:=\sqrt{-p_{\tau}}=\sqrt{K \gamma} \tau^{-\frac{\gamma+1}{2}} e^{\frac{S}{2 c_{\tau}}} .
$$

\subsection{Coordinates}

We use the coordinates introduced by Temple and Young in [25]. Define new variables $m$ and $\eta$ for $S$ and $\tau$, by

$$
m:=e^{\frac{S}{2 c_{\tau}}}>0
$$

and, referring to (2.7),

$$
\eta:=\int_{\tau}^{\infty} \frac{c}{m} d \tau=\frac{2 \sqrt{K \gamma}}{\gamma-1} \tau^{-\frac{\gamma-1}{2}}>0
$$

It follows that

$$
\begin{aligned}
\tau & =K_{\tau} \eta^{-\frac{2}{\gamma-1}} \\
p & =K_{p} m^{2} \eta^{\frac{2 \gamma}{\gamma-1}} \\
c & =c(\eta, m)=K_{c} m \eta^{\frac{\gamma+1}{\gamma-1}},
\end{aligned}
$$


where $K_{\tau}, K_{p}$ and $K_{c}$ are positive constants given by

$$
K_{\tau}=\left(\frac{2 \sqrt{K \gamma}}{\gamma-1}\right)^{\frac{2}{\gamma-1}}, \quad K_{p}=K K_{\tau}^{-\gamma}, \quad \text { and } \quad K_{c}=\sqrt{K \gamma} K_{\tau}^{-\frac{\gamma+1}{2}},
$$

so that also

$$
K_{p}=\frac{\gamma-1}{2 \gamma} K_{c} \quad \text { and } \quad K_{\tau} K_{c}=\frac{\gamma-1}{2} .
$$

In these coordinates, for $C^{1}$ solutions, equations (2.1) $-(2.3)$ are equivalent to

$$
\begin{aligned}
\eta_{t}+\frac{c}{m} u_{x} & =0, \\
u_{t}+m c \eta_{x}+2 \frac{p}{m} m_{x} & =0, \\
m_{t} & =0,
\end{aligned}
$$

the last equation being (2.5), which is equivalent to (2.3). Note that, while the solution remains $C^{1}, m=m(x)$ is given by the initial data and can be regarded as stationary.

\section{2 $\quad L^{\infty}$ bounds for $C^{1}$ solutions}

Our first goal is to provide a uniform upper bound for the density $\rho$ and velocity $|u|$, depending only on the initial data, for $C^{1}$ solutions of (2.1)(2.3). Denote the Riemann invariants by

$$
r:=u-m \eta, \quad s:=u+m \eta .
$$

For isentropic $C^{1}$ solutions ( $m$ constant) the Riemann invariants $s$ and $r$ are constants along forward and backward characteristics, respectively. However, for general non-isentropic flow, $s$ and $r$ vary along characteristics.

The forward and backward characteristics are described by

$$
\frac{d x}{d t}=c \quad \text { and } \quad \frac{d x}{d t}=-c,
$$

and we denote the corresponding directional derivatives along these by

$$
\partial_{+}:=\frac{\partial}{\partial t}+c \frac{\partial}{\partial x} \quad \text { and } \quad \partial_{-}:=\frac{\partial}{\partial t}-c \frac{\partial}{\partial x},
$$

respectively. Using (2.16), (2.10) and (2.12), equations (2.13) and (2.14) become

$$
\begin{aligned}
& \partial_{+} s=\frac{1}{2 \gamma} \frac{\partial_{+} m}{m}(s-r), \\
& \partial_{-} r=\frac{1}{2 \gamma} \frac{\partial_{-} m}{m}(r-s),
\end{aligned}
$$


while by (2.3), we have

$$
\partial_{+} m=c m^{\prime}(x), \quad \text { and } \quad \partial_{-} m=-c m^{\prime}(x) .
$$

Applying an integrating factor and setting

$$
\widetilde{s}=m^{-\frac{1}{2 \gamma}} s \quad \text { and } \quad \widetilde{r}=m^{-\frac{1}{2 \gamma}} r,
$$

we obtain the system

$$
\begin{aligned}
& \partial_{+} \widetilde{s}=-\frac{1}{2 \gamma} \frac{\partial_{+} m}{m} \widetilde{r}, \\
& \partial_{-} \widetilde{r}=-\frac{1}{2 \gamma} \frac{\partial_{-} m}{m} \widetilde{s} .
\end{aligned}
$$

We obtain upper bounds for $C^{1}$ solutions of (2.1)-(2.3) by bounding $|\widetilde{s}|$ and $|\widetilde{r}|$. We assume that the initial entropy $S(x)$ is $C^{1}$ and has finite total variation, so that

$$
V:=\frac{1}{2 c_{\tau}} \int_{-\infty}^{+\infty}\left|S^{\prime}(x)\right| d x=\int_{-\infty}^{+\infty} \frac{\left|m^{\prime}(x)\right|}{m(x)} d x<\infty
$$

while also, by (2.8),

$$
0<M_{L}<m(\cdot)<M_{U}
$$

for some constants $M_{L}$ and $M_{U}$. Also, since $\rho$ and $|u|$ are bounded initially, there exist positive constants $M_{s}$ and $M_{r}$, such that, in the initial data,

$$
\left|\widetilde{s}_{0}(\cdot)\right|<M_{s} \text { and }\left|\widetilde{r}_{0}(\cdot)\right|<M_{r} .
$$

We define two useful constants by

$$
\begin{aligned}
& N_{1}:=M_{s}+\bar{V} M_{r}+\bar{V}\left(\bar{V} M_{s}+\bar{V}^{2} M_{r}\right) e^{\bar{V}^{2}}, \\
& N_{2}:=M_{r}+\bar{V} M_{s}+\bar{V}\left(\bar{V} M_{r}+\bar{V}^{2} M_{s}\right) e^{\bar{V}^{2}},
\end{aligned}
$$

where $\bar{V}:=\frac{V}{2 \gamma}$, which clearly depend only on the initial data.

Theorem 2.1. For $C^{1}$ solutions of (2.1)-(2.3), we have the a priori bounds

$$
|u| \leq \frac{N_{1}+N_{2}}{2} M_{U}^{\frac{1}{2 \gamma}} \quad \text { and } \quad \rho \leq \frac{N_{1}+N_{2}}{2} M_{L}^{\frac{1}{2 \gamma}-1},
$$

Note that the density $\rho$ need not be bounded away from zero, as vacuums can form in infinite time [26, 5]. The theorem is an immediate consequence of the following estimate. 
Lemma 2.2. For a given point $\left(x_{1}, t_{1}\right)$, suppose the solution is $C^{1}$ in the characteristic triangle bounded by the forward and backward characteristics through $\left(x_{1}, t_{1}\right)$ and the line $t=0$. Then

$$
\left|\widetilde{s}\left(x_{1}, t_{1}\right)\right| \leq N_{1} \quad \text { and } \quad\left|\widetilde{r}\left(x_{1}, t_{1}\right)\right| \leq N_{2} .
$$

Proof. We prove the bound on $\widetilde{s}\left(x_{1}, t_{1}\right)$; the bound of $\widetilde{r}\left(x_{1}, t_{1}\right)$ is obtained similarly. Referring to Figure 1, we denote the forward and backward characteristics through a point $\left(x_{*}, t_{*}\right)$ by

$$
\begin{aligned}
& \overrightarrow{\mathcal{L}}_{x_{*}}=\left\{(x, \vec{t}(x)) \mid x \leq x_{*}\right\}=\left\{(\vec{x}(t), t) \mid t \leq t_{*}\right\} \quad \text { and } \\
& \overleftarrow{\mathcal{L}}_{x_{*}}=\left\{(x, \overleftarrow{t}(x)) \mid x \geq x_{*}\right\}=\left\{(\overleftarrow{x}(t), t) \mid t \leq t_{*}\right\}
\end{aligned}
$$

respectively, parameterized by $x$ or $t$, as convenient.

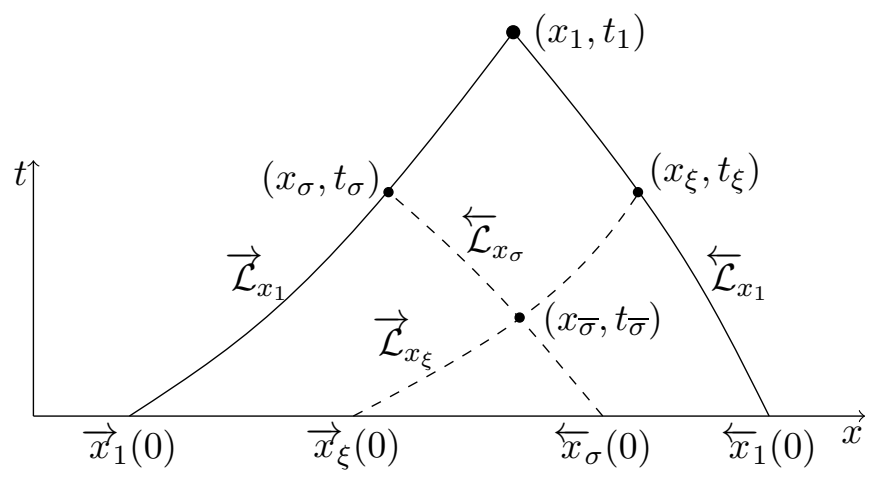

Figure 1: Characteristic triangle

We integrate (2.20) along the forward characteristic $\overrightarrow{\mathcal{L}}_{x_{1}}$ from $\left(\vec{x}_{1}(0), 0\right)$ to $\left(x_{1}, t_{1}\right)$, with a change of integration variable, and use $x_{\sigma}$ as the new parameter. We then integrate (2.21) along the backward characteristics $\overleftarrow{\mathcal{L}}_{x_{\sigma}}$, from 0 to $t_{\sigma}$, for each $\left(x_{\sigma}, t_{\sigma}\right) \in \overrightarrow{\mathcal{L}}_{x_{1}}$, and again change variables. We have

$$
\widetilde{s}\left(x_{1}, t_{1}\right)=\widetilde{s}_{0}\left(\vec{x}_{1}(0)\right)-\frac{1}{2 \gamma} \int_{\vec{x}_{1}(0)}^{x_{1}} \frac{m^{\prime}}{m}\left(x_{\sigma}\right) \widetilde{r}\left(x_{\sigma}, \vec{t}\left(x_{\sigma}\right)\right) d x_{\sigma},
$$

where we have used (2.19), and similarly

$$
\widetilde{r}\left(x_{\sigma}, t_{\sigma}\right)=\widetilde{r}_{0}\left(\overleftarrow{x}_{\sigma}(0)\right)-\frac{1}{2 \gamma} \int_{\overleftarrow{x}_{\sigma}(0)}^{x_{\sigma}} \frac{m^{\prime}}{m}(x) \widetilde{s}\left(x, \overleftarrow{t}_{\sigma}(x)\right) d x
$$


Here $\mathcal{Y}$ and $\mathcal{X}$ indicate the direction of the characteristic along which the integration is performed. Combining these, we get

$$
\begin{aligned}
\widetilde{s}\left(x_{1}, t_{1}\right)=\widetilde{s}_{0}\left(\vec{x}_{1}(0)\right)-\frac{1}{2 \gamma} \underset{\vec{x}_{1}(0)}{f_{1}^{x_{1}}} \frac{m^{\prime}}{m}\left(x_{\sigma}\right) \widetilde{r}_{0}\left(\overleftarrow{x}_{\sigma}(0)\right) d x_{\sigma} \\
+\frac{1}{4 \gamma^{2}} \underset{\vec{x}_{1}(0)}{f_{1}} \frac{m^{\prime}}{m}\left(x_{\sigma}\right)\left(\int_{\overleftarrow{x}_{\sigma}(0)}^{x_{\sigma}} \frac{m^{\prime}}{m}(x) \widetilde{s}\left(x, \overleftarrow{t}_{\sigma}(x)\right) d x\right) d x_{\sigma} .
\end{aligned}
$$

The first two terms can be estimated by our initial bounds, and we will apply a Gronwall inequality to estimate the third term.

Equation (2.25) continues to hold for any point $\left(x_{\xi}, t_{\xi}\right)$ on the backward characteristic $\overleftarrow{\mathcal{L}}_{x_{1}}$, with $x_{1}$ replaced by $x_{\xi}$. We multiply (2.25) by $\frac{\partial_{+} m}{m}\left(x_{\xi}\right)$ take absolute values, integrate in time along the backward characteristic $\overleftarrow{\mathcal{L}}_{x_{1}}$, and change to spatial variables. Doing this, we get

$$
\begin{aligned}
& \left.\int_{x_{1}}^{\overleftarrow{x}_{1}(0)} \mid \widetilde{s}\left(x_{\xi}, t_{\xi}\right)\right)\left|d \mu\left(x_{\xi}\right) \leq \int_{x_{1}}^{\overleftarrow{x}_{1}(0)}\right| \widetilde{s}_{0}\left(\vec{x}_{\xi}(0)\right) \mid d \mu\left(x_{\xi}\right) \\
& +\frac{1}{2 \gamma} \int_{x_{1}}^{\overleftarrow{x}_{1}(0)} \int_{\vec{x}_{\xi}(0)}^{x_{\xi}}\left|\widetilde{r}_{0}\left(\overleftarrow{x}_{\bar{\sigma}}(0)\right)\right| d \mu\left(x_{\bar{\sigma}}\right) d \mu\left(x_{\xi}\right) \\
& +\frac{1}{4 \gamma^{2}} \int_{x_{1}}^{\overleftarrow{x}_{1}(0)} \int_{\vec{x}_{\xi}(0)}^{x_{\xi}} \overleftarrow{x}_{\bar{x}_{\bar{\sigma}}(0)}\left|\widetilde{s}\left(x, \overleftarrow{t}_{\bar{\sigma}}(x)\right)\right| d \mu(x) d \mu\left(x_{\bar{\sigma}}\right) d \mu\left(x_{\xi}\right)
\end{aligned}
$$

where $d \mu$ is the Stieltjes measure

$$
d \mu(x):=\left|\frac{m^{\prime}}{m}(x)\right| d x
$$

which has total mass at most $V$ along any characteristic. Again the first two terms of (2.26) can be estimated by the initial data, so we focus on the last term.

For $\left(x_{\sigma}, t_{\sigma}\right)$ on the forward characteristic $\overrightarrow{\mathcal{L}}_{x_{1}}$, set

$$
F\left(x_{\sigma}\right):=\int_{x_{\sigma}}^{\overleftarrow{x}_{\sigma}(0)}\left|\widetilde{s}\left(x, \overleftarrow{t}_{\sigma}(x)\right)\right| d \mu(x)
$$


where the integral is along the backward characteristic $\overleftarrow{\mathcal{L}}_{x_{\sigma}}$. If the backward characteristic through $\left(x, \overleftarrow{t}_{\bar{\sigma}}(x)\right)$ is extended up to $\overrightarrow{\mathcal{L}}_{x_{1}}$, the innermost integral in (2.26) is estimated by

$$
\int_{x_{\bar{\sigma}}}^{\overleftarrow{x}_{\bar{\sigma}}(0)}|\widetilde{s}(x, \overleftarrow{t} \bar{\sigma}(x))| d \mu(x) \leq F\left(x_{\sigma}\right)
$$

provided $\left(x_{\bar{\sigma}}, t_{\bar{\sigma}}\right) \in \overleftarrow{\mathcal{L}}_{x_{\sigma}}$, so that also $\overleftarrow{x}_{\bar{\sigma}}(0)=\overleftarrow{x}_{\sigma}(0)$, and we change the order of the other two integrations. Using (2.27) with (2.22) and (2.24), it follows that (2.26) yields

$$
\begin{aligned}
F\left(x_{1}\right) & \leq V M_{s}+\frac{1}{2 \gamma} V^{2} M_{r}+\frac{1}{4 \gamma^{2}} \int_{\vec{x}_{1}(0)}^{x_{1}} \int_{x_{\sigma}}^{\overleftarrow{x}_{\sigma}(0)} F\left(x_{\sigma}\right) d \mu(\bar{x}) d \mu\left(x_{\sigma}\right) \\
& \leq V M_{s}+\frac{1}{2 \gamma} V^{2} M_{r}+\frac{1}{4 \gamma^{2}} V{\underset{x_{1}(0)}{x_{1}}}_{f} F\left(x_{\sigma}\right) d \mu\left(x_{\sigma}\right) .
\end{aligned}
$$

We now use Gronwall's inequality to get

$$
F\left(x_{1}\right) \leq\left(V M_{s}+\frac{1}{2 \gamma} V^{2} M_{r}\right) e^{\frac{V^{2}}{4 \gamma^{2}}}=: \hat{N}_{1} .
$$

It follows similarly that for $\left(x_{\sigma}, t_{\sigma}\right) \in \overrightarrow{\mathcal{L}}_{x_{1}}$, we have $F\left(x_{\sigma}\right) \leq \hat{N}_{1}$. Finally, using this estimate in (2.25), we get

$$
\left|\widetilde{s}\left(x_{1}, t_{1}\right)\right| \leq M_{s}+\frac{1}{2 \gamma} V M_{r}+\frac{1}{4 \gamma^{2}} V \hat{N}_{1}=N_{1},
$$

and the proof is complete.

\subsection{Singularity formation}

In [2], we introduce gradient variables

$$
\begin{aligned}
& y=m^{-\frac{3(3-\gamma)}{2(3 \gamma-1)}} \eta^{\frac{\gamma+1}{2(\gamma-1)}}\left((u+m \eta)_{x}-\frac{2}{3 \gamma-1} m_{x} \eta\right) \quad \text { and } \\
& q=m^{-\frac{3(3-\gamma)}{2(3 \gamma-1)}} \eta^{\frac{\gamma+1}{2(\gamma-1)}}\left((u-m \eta)_{x}+\frac{2}{3 \gamma-1} m_{x} \eta\right),
\end{aligned}
$$

and derive Riccati type equations for their evolution. 
Lemma 2.3. [2] For $C^{2}$ solutions of (2.1)-(2.3), we have

$$
\begin{aligned}
& \partial_{+} y=a_{0}+a_{2} y^{2}, \\
& \partial_{-} q=a_{0}+a_{2} q^{2},
\end{aligned}
$$

where

$$
\begin{aligned}
& a_{0}=\frac{K_{c}}{\gamma}\left[\frac{\gamma-1}{3 \gamma-1} m m_{x x}-\frac{(3 \gamma+1)(\gamma-1)}{(3 \gamma-1)^{2}} m_{x}^{2}\right] m^{-\frac{3(3-\gamma)}{2(3 \gamma-1)}} \eta^{\frac{3(\gamma+1)}{2(\gamma-1)}+1}, \\
& a_{2}=-K_{c} \frac{\gamma+1}{2(\gamma-1)} m^{\frac{3(3-\gamma)}{2(3 \gamma-1)}} \eta^{\frac{\gamma+1}{2(\gamma-1)}-1}<0
\end{aligned}
$$

Furthermore,

$$
|y| \text { or }|q| \rightarrow \infty \quad \text { iff } \quad\left|u_{x}\right| \text { or }\left|\tau_{x}\right| \rightarrow \infty
$$

Similar equations are derived independently in [18]. Using these equations, a singularity formation result was proved in 2]. By applying Lemma 2.2, we can improve that result to obtain our main theorem on the Euler equations.

Theorem 2.4. Assume the initial data are $C^{2}$ with entropy having bounded variation, and suppose there is a positive constant $M_{*}$ such that the initial entropy satisfies $\left|m^{\prime \prime}(x)\right|<M_{*}$. If $1<\gamma<3$, we also assume that the density has a positive global lower bound. There exists positive constant $N$ depending only on the initial data, such that, if the initial data satisfies

$$
\inf \{y(\cdot, 0), q(\cdot, 0)\}<-N,
$$

then $\left|u_{x}\right|$ and/or $\left|\tau_{x}\right|$ blow up in finite time.

This result is an extension of Lax's singularity formation result in [13] for $2 \times 2$ strictly hyperbolic systems. We recover the isentropic case by taking $m$ constant, so $a_{0}=0$. The conditions which guarantee blowup are expressed in terms of global bounds for the variables, so we must ensure that the state does not leave some compact set, and, in particular, the vacuum must be avoided. When $1<\gamma<3$, our estimates depend on a lower bound for the density as well as on the upper bound. This is also true in the isentropic case treated by Lax. However, when $1<\gamma<3$, we expect a more refined analysis to show that even if the solution approaches vacuum, the gradient still blows up in finite time [29].

Proof. By Theorem 2.1 and (2.9), we know $\eta$ has a global upper bound depending only on the initial data, denoted $E_{U}$. 
By (2.30), it is easy to calculate that, if $a_{0} \geq 0$,

$$
\sqrt{-\frac{a_{0}}{a_{2}}}=\sqrt{\frac{2(\gamma-1)^{2}}{\gamma(\gamma+1)(3 \gamma-1)}\left(m m_{x x}-\frac{3 \gamma+1}{3 \gamma-1} m_{x}^{2}\right)} \eta^{\frac{\gamma+1}{2(\gamma-1)}+1} m^{-\frac{3(3-\gamma)}{2(3 \gamma-1)}},
$$

which implies the uniform bound $\sqrt{-a_{0} / a_{2}} \leq N$, where

$$
N:= \begin{cases}\sqrt{\frac{2(\gamma-1)^{2}}{\gamma(\gamma+1)(3 \gamma-1)} M_{*}} E_{U}^{\frac{3 \gamma-1}{2(\gamma-1)}} M_{L}^{\frac{3 \gamma-5}{3 \gamma-1}}, & 1<\gamma \leq 5 / 3, \\ \sqrt{\frac{2(\gamma-1)^{2}}{\gamma(\gamma+1)(3 \gamma-1)} M_{*}} E_{U}^{\frac{3 \gamma-1}{2(\gamma-1)}} M_{U}^{\frac{3 \gamma-5}{3 \gamma-1}}, & \gamma \geq 5 / 3 .\end{cases}
$$

It follows that if $y<-N$, then $a_{0}+a_{2} y^{2}<0$.

Now suppose that (2.32) holds. Then there exist $\varepsilon>0$ and $x_{0}$ such that

$$
y\left(x_{0}, 0\right)<-(1+\varepsilon) N,
$$

say. Now consider the forward characteristic starting at $\left(x_{0}, 0\right)$. By (2.29), along this characteristic (parametrized by $t$ ) we have $\partial_{+} y<0$, so also

$$
y(t)<-(1+\varepsilon) N \quad \text { for } \quad t \geq 0,
$$

which in turn implies

$$
a_{0}+a_{2} \frac{y(t)^{2}}{(1+\varepsilon)^{2}}<0
$$

for all $t \geq 0$. Now (2.29) implies

$$
\partial_{+} y=a_{0}+a_{2} y^{2}<\left(1-\frac{1}{(1+\varepsilon)^{2}}\right) a_{2} y^{2}<0,
$$

since $a_{2}<0$. Integrating, we get

$$
\frac{1}{y(t)} \geq \frac{1}{y(0)}-f_{0}^{t}\left(1-\frac{1}{(1+\varepsilon)^{2}}\right) a_{2} d t
$$

where the integral is along the forward characteristic. By (2.30), when $\gamma \geq 3, a_{2}$ is negative and bounded above, so the right hand side of (2.34) approaches zero in finite time. This implies that $y(t)$ approaches $-\infty$ in finite time, so that $\left|\tau_{x}\right|$ and/or $\left|u_{x}\right|$ blow up.

When $1<\gamma<3$, and assuming $\eta$ has a positive (global) lower bound along the characteristic, then $a_{2}$ is again negative and bounded above, so the result follows similarly. It is clear that the same argument holds along a backward characteristic if inf $q<-N$. 


\section{Magnetohydrodynamics}

\subsection{Prior results for generalized Euler equations}

We recall the results of [4] for the generalized $p$-system (which includes the smooth Euler equations) in one space dimension,

$$
\begin{aligned}
\tau_{t}-u_{x} & =0, \\
u_{t}+P(\tau, x)_{x} & =0,
\end{aligned}
$$

where $P(\tau, x)$ is a $C^{3}$ function of $\tau>0$ and $x$, satisfying

$$
P_{\tau}<0 \text { and } P_{\tau \tau}>0
$$

so that the system is hyperbolic with wavespeed

$$
c:=c(\tau, x)=\sqrt{-P_{\tau}} .
$$

It is convenient to introduce new variables $(h, \mu)$ for $(\tau, x)$, by setting

$$
h(\tau, x):=\int_{\tau}^{\tau^{*}} c d \tau=\int_{\tau}^{\tau^{*}} \sqrt{-P_{\tau}} d \tau \text { and } \mu=x
$$

where $\tau^{*}$ is a constant or infinity. It follows that

$$
h_{\tau}=-c, \quad \tau_{h}=-\frac{1}{c}, \quad \text { and } \quad P_{h}=c,
$$

and furthermore, for any $C^{1}$ function $f(\tau, x)$,

$$
f_{h}=-\frac{f_{\tau}}{c} \quad \text { and } \quad f_{\mu}=\frac{h_{x}}{c} f_{\tau}+f_{x} .
$$

We define gradient variables by

$$
\begin{aligned}
y & :=\sqrt{c}(u+h)_{x}+\frac{P_{\mu}}{\sqrt{c}}-I, \\
q & :=\sqrt{c}(u-h)_{x}-\frac{P_{\mu}}{\sqrt{c}}+I,
\end{aligned}
$$

where

$$
I=I(h, \mu)=\int_{h_{0}}^{h} \frac{1}{2} \sqrt{c}\left(\frac{P_{\mu}}{c}\right)_{h} d h,
$$

and $h_{0}$ is a constant. 
Lemma 3.1. [4] For $C^{2}$ solutions of (3.1), we have

$$
\begin{aligned}
& \partial_{+} y=a_{0}+a_{1} y+a_{2} y^{2}, \\
& \partial_{-} q=a_{0}-a_{1} q+a_{2} q^{2},
\end{aligned}
$$

where

$$
\begin{aligned}
& a_{0}=-c I_{\mu}+\frac{1}{2} \sqrt{c}\left(\frac{P_{\mu}}{c}\right)_{h} P_{\mu}-c\left(\frac{P_{\mu}}{c}\right)_{h} I-\frac{c_{h}}{2 \sqrt{c}} I^{2}, \\
& a_{1}=-(2 \sqrt{c} I)_{h}=-c\left(\frac{P_{\mu}}{c}\right)_{h}-\frac{c_{h}}{\sqrt{c}} I, \\
& a_{2}=-\frac{c_{h}}{2 \sqrt{c}}<0 .
\end{aligned}
$$

For arbitrarily given positive constants $A_{i}$ and $B_{i}$, we denote by $\mathcal{K}$ the compact set whose interior $\mathcal{K}^{o}$ is given by

$$
\begin{gathered}
|h|<B_{1}, \quad A_{2}<c<B_{2}, \quad A_{3}<c_{h}<B_{3}, \quad\left|c_{\mu}\right|<B_{4} \\
\left|c_{\mu \mu}\right|<B_{5}, \quad\left|c_{h \mu}\right|<B_{6}, \quad\left|p_{\mu}\right|<B_{7}, \quad\left|p_{\mu \mu}\right|<B_{8} .
\end{gathered}
$$

Lemma 3.2. [4] There exists a constant $\widetilde{N}>0$ depending only on $\mathcal{K}$, such that, if the $C^{2}$ initial data of (3.1) satisfy

$$
(u(x, 0), \tau(x, 0)) \in \mathcal{K}^{o}, \quad \text { for all } x,
$$

and if

$$
\inf \{y(\cdot, 0), q(\cdot, 0)\}<-\widetilde{N},
$$

then there exists $T_{*}=T_{*}(\mathcal{K}, \widetilde{N})$ such that either

$$
\max \left\{\left|u_{x}\right|,\left|\tau_{x}\right|\right\} \rightarrow \infty \quad \text { as } t \rightarrow T_{*},
$$

or there is some point $\left(x_{b}, t_{b}\right)$ with $t_{b} \leq T_{*}$ such that

$$
\left(u\left(x_{b}, t_{b}\right), \tau\left(x_{b}, t_{b}\right)\right) \in \partial \mathcal{K} .
$$

\subsection{Refinements for MHD}

In this section, we apply and upgrade Lemma 3.2 for one-D Magnetohydrodynamics. The system models the motion of a smooth compressible fluid 
coupled to a magnetic field $H=\left(H_{1}, H_{2}, H_{3}\right)$. In a Lagrangian frame in one space dimension, $C^{1}$ solutions satisfy the quasilinear system

$$
\begin{aligned}
\frac{\partial \tau}{\partial t}-\frac{\partial u_{1}}{\partial x} & =0, \\
\frac{\partial H_{j}}{\partial t}+\rho H_{j} \frac{\partial u_{1}}{\partial x}-\rho H_{1} \frac{\partial u_{j}}{\partial x} & =0, \quad j=2,3 \\
\frac{\partial u_{1}}{\partial t}+\frac{\partial}{\partial x}\left(p+\frac{1}{2} \mu_{0}\left(H_{2}^{2}+H_{3}^{2}\right)\right) & =0, \\
\frac{\partial u_{j}}{\partial t}-\mu_{0} H_{1} \frac{\partial H_{j}}{\partial x} & =0, \quad j=2,3 \\
\frac{\partial S}{\partial t} & =0,
\end{aligned}
$$

Here the fluid quantities are the density $\rho$, specific volume $\tau=\rho^{-1}$, velocity field $\left(u_{1}, u_{2}, u_{3}\right)$, entropy $S$ and hydrostatic pressure $p=p(\tau, S)$, while $\left(H_{1}, H_{2}, H_{3}\right)$ is the magnetic field and $\mu_{0}$ is the magnetic constant [17]. In one-dimensional MHD, the first component $H_{1}$ of the magnetic field is necessarily constant.

System (3.8) simplifies significantly if we take the constant $H_{1}$ to vanish, so the magnetic field is perpendicular to the direction of motion. In this case, the full system (3.8) can be written

$$
\begin{aligned}
& \frac{\partial \tau}{\partial t}-\frac{\partial u_{1}}{\partial x}=0, \\
& \frac{\partial u_{1}}{\partial t}+\frac{\partial \widetilde{p}}{\partial x}=0
\end{aligned}
$$

regarded as a generalized $p$-system, and coupled with

$$
\frac{\partial \widetilde{H}_{j}}{\partial t}=0, \quad \frac{\partial u_{j}}{\partial t}=0, \quad \frac{\partial S}{\partial t}=0, \quad j=1,2,
$$

where we have written

$$
\widetilde{H}_{j}:=\tau H_{j} \quad \text { and } \quad \widetilde{p}:=p+\frac{1}{2} \mu_{0} \frac{\widetilde{H}_{2}^{2}+\widetilde{H}_{3}^{2}}{\tau^{2}} .
$$

System (3.9) is clearly an example of (3.1), so all results in [4] apply directly. Here we obtain a stronger singularity formation result than Lemma 3.2 by restricting our consideration to a polytropic ideal gas,

$$
p=K e^{\frac{S}{c \tau}} \tau^{-\gamma}
$$

with adiabatic gas constant $1<\gamma \leq 2$. 
Theorem 3.3. For system (3.9), (3.10), assume that the initial data is $C^{2}$ with uniform bounds

$$
|G(\cdot, 0)|<N_{0}, \quad\left|G(\cdot, 0)_{x}\right|<N_{1}, \quad \text { and } \quad\left|G(\cdot, 0)_{x x}\right|<N_{2}
$$

for each of $G=S, H_{2}, H_{3}$. Assume also that the density is globally bounded,

$$
0<N_{\rho L}<\rho(x, t)<N_{\rho U}, \quad \text { for any }(x, t) \in \mathbb{R} \times \mathbb{R}^{+},
$$

for constants $N_{\rho L}$ and $N_{\rho U}$. There exists a positive constant $\widehat{N}$, depending on $N_{j}$ and $N_{\rho U}$, but not on $N_{\rho L}$, such that, if

$$
\inf \{y(\cdot, 0), q(\cdot, 0)\}<-\widehat{N},
$$

then $u_{x}$ and/or $\tau_{x}$ blow up in finite time. Here $y$ and $q$ are defined in (3.4), with $P=\widetilde{p}$.

Although we assume the global bounds (3.11), $\widehat{N}$ is independent of the lower bound $N_{\rho L}$ of density. Theorem 2.1 for the compressible Euler equations indicates that it is reasonable to expect an a priori upper bound on the density, and such a bound would imply that $\widehat{N}$ depends only on the initial data.

Proof. As in the proof of Theorem 2.4, from (3.6), it suffices to find uniform bounds for the roots of the quadratic equations

$$
a_{0}+a_{1} y+a_{2} y^{2}=0 \quad \text { and } \quad a_{0}-a_{1} q+a_{2} q^{2}=0,
$$

whose coefficients are given in (3.7) with $P$ replaced by $\widetilde{p}$. That is, we must choose $\widehat{N}$ so that

$$
\left|\frac{a_{1}}{2 a_{2}}\right|+\sqrt{\left|\frac{a_{1}}{2 a_{2}}\right|^{2}+\frac{a_{0}}{\left|a_{2}\right|}} \leq \widehat{N}
$$

when $a_{1}^{2}-4 a_{0} a_{2} \geq 0$, where we calculate

$$
\left|\frac{a_{1}}{2 a_{2}}\right|=\left|\frac{c \sqrt{c}}{c_{h}}\left(\frac{\widetilde{p}_{\mu}}{c}\right)_{h}+I\right|,
$$

and

$$
\left|\frac{a_{1}}{2 a_{2}}\right|^{2}+\frac{a_{0}}{\left|a_{2}\right|}=\left(\frac{c \sqrt{c}}{c_{h}}\left(\frac{\widetilde{p}_{\mu}}{c}\right)_{h}\right)^{2}-2 \frac{c \sqrt{c}}{c_{h}} I_{\mu}+\frac{c}{c_{h}}\left(\frac{\widetilde{p}_{\mu}}{c}\right)_{h} \widetilde{p}_{\mu} .
$$


It follows that we can choose $\widehat{N}$ provided we find upper bounds (independent of $N_{\rho L}$ ) for each of the quantities

$$
\left|\left(\frac{\tilde{p}_{\mu}}{c}\right)_{h}\right|, \quad\left|\frac{\tilde{p}_{\mu}}{\sqrt{c}}\right|, \quad\left|\frac{c \sqrt{c}}{c_{h}}\right|, \quad|I|, \quad \text { and } \quad\left|I_{\mu}\right| ;
$$

we treat each of these terms separately.

In order to simplify the calculation, we denote

$$
A(x):=K e^{\frac{S}{c_{\tau}}}, \quad B(x):=\frac{1}{2} \mu_{0}\left(\widetilde{H}_{2}^{2}+\widetilde{H}_{3}^{2}\right),
$$

so that $A(x)$ and $B(x)$ are positive bounded functions, and $A(x)$ is bounded from below by a positive constant. With this notation,

$$
\widetilde{p}(\tau, x)=A(x) \tau^{-\gamma}+B(x) \tau^{-2},
$$

the wave speed is

$$
c=\sqrt{-\widetilde{p}_{\tau}}=\sqrt{\gamma A \tau^{-\gamma-1}+2 B \tau^{-3}},
$$

and

$$
h=\int_{\tau}^{\infty} c d \tau=\int_{\tau}^{\infty} \sqrt{\gamma A \tau^{-\gamma-1}+2 B \tau^{-3}} d \tau .
$$

It follows by (3.11) that $\widetilde{p}, c$ and $h$ are bounded above and below. We write

$$
0<N_{h L}<h<N_{h U},
$$

where $N_{h U}$ does not depend on $N_{\rho L}$.

1. We first consider $\left(\frac{\widetilde{p}_{\mu}}{c}\right)_{h}$. By (3.2) we have $\widetilde{p}_{\mu h}=c_{\mu}$, and by (3.3),

$$
c\left(\frac{\widetilde{p}_{\mu}}{c}\right)_{h}=c_{\mu}-c_{h} \frac{\widetilde{p}_{\mu}}{c}=c_{x}+\frac{c_{\tau}}{c^{2}} \widetilde{p}_{x}=\frac{c}{2}\left(\frac{\widetilde{p}_{x}}{\widetilde{p}_{\tau}}\right)_{\tau},
$$

and thus

$$
\left(\frac{\widetilde{p}_{\mu}}{c}\right)_{h}=\frac{1}{2}\left(\frac{\widetilde{p}_{x}}{\widetilde{p}_{\tau}}\right)_{\tau}=\frac{\widetilde{p}_{x \tau} \widetilde{p}_{\tau}-\widetilde{p}_{x} \widetilde{p}_{\tau \tau}}{2 \widetilde{p}_{\tau}^{2}} .
$$

Now, since $1<\gamma \leq 2$, we have

$$
\left|\widetilde{p}_{\tau}\right|=\gamma A \tau^{-\gamma-1}+2 B \tau^{-3} \geq \gamma A \tau^{-\gamma-1},
$$

and since $A$ is bounded from below, $\left(\frac{\widetilde{p}_{\mu}}{c}\right)_{h}$ is bounded provided

$$
F_{1}=\left(\widetilde{p}_{x \tau} \widetilde{p}_{\tau}-\widetilde{p}_{x} \widetilde{p}_{\tau \tau}\right) \tau^{2 \gamma+2}
$$


is bounded. It is routine to compute

$F_{1}=\left(\gamma A_{x}+2 B_{x} \tau^{\gamma-2}\right)\left(\gamma A+2 B \tau^{\gamma-2}\right)-\left(A_{x}+B_{x} \tau^{\gamma-2}\right)\left(\gamma(\gamma+1) A+6 B \tau^{\gamma-2}\right)$, which is bounded independent of $N_{\rho L}$ for $\gamma \leq 2$.

2. By (3.3),

$$
\frac{\widetilde{p}_{\mu}}{\sqrt{c}}=\frac{\widetilde{p}_{x}}{\sqrt{c}}+h_{x} \frac{\widetilde{p}_{\tau}}{c \sqrt{c}}=\frac{\widetilde{p}_{x}}{\sqrt{c}}-\sqrt{c} h_{x}
$$

and this is bounded by a constant independent of $N_{\rho L}$, since

$$
\frac{\widetilde{p}_{x}}{\sqrt{c}}=\frac{A_{x} \tau^{-\gamma}+B_{x} \tau^{-2}}{\left(\gamma A \tau^{-\gamma-1}+2 B \tau^{-3}\right)^{\frac{1}{4}}}=O(1) \tau^{-\frac{3 \gamma-1}{4}},
$$

and

$$
h_{x}=\int_{\tau}^{\infty} \frac{\gamma A_{x} \tau^{-\frac{\gamma+1}{2}}+2 B_{x} \tau^{\frac{\gamma-5}{2}}}{2 \sqrt{\gamma A+2 B \tau^{\gamma-2}}} d \tau=O(1) \tau^{-\frac{\gamma-1}{2}}
$$

so $\sqrt{c} h_{x}=O(1) \tau^{-\frac{3 \gamma-1}{4}}$, where $O(1)$ is a bound depending on $x$ and the lower bound for $\tau$, and thus is independent of $N_{\rho L}$.

3. We similarly calculate

$$
\frac{c \sqrt{c}}{c_{h}}=\frac{c \sqrt{c}}{c_{\tau} \tau_{h}}=-\frac{2 c^{\frac{7}{2}}}{2 c c_{\tau}}=\frac{2\left(\gamma A \tau^{-\gamma-1}+2 B \tau^{-3}\right)^{\frac{7}{4}}}{\gamma(\gamma+1) A \tau^{-\gamma-2}+6 B \tau^{-4}}=O(1) \tau^{-\frac{3 \gamma-1}{4}},
$$

which is again bounded independent of $N_{\rho L}$.

4. Since the upper bounds of $c, h$ and $\left|\left(\frac{\widetilde{p}_{\mu}}{c}\right)_{h}\right|$ don't depend on $N_{\rho L}$, the absolute value of $I$ is bounded above by a constant independent of $N_{\rho L}$.

5. By (3.5), (3.3),

$$
I_{\mu}=\int_{h_{0}}^{h}\left(\frac{1}{2} \sqrt{c}\left(\frac{\widetilde{p}_{\mu}}{c}\right)_{h}\right)_{\mu} d h=\int_{h_{0}}^{h}\left[\frac{c_{\mu}}{4 \sqrt{c}}\left(\frac{\widetilde{p}_{\mu}}{c}\right)_{h}+\frac{\sqrt{c}}{2}\left(\frac{\widetilde{p}_{\mu}}{c}\right)_{h \mu}\right] d h,
$$

so it suffices to bound $\frac{c_{\mu}}{\sqrt{c}}$ and $\sqrt{c}\left(\frac{\widetilde{p}_{\mu}}{c}\right)_{h \mu}$. By (3.3),

$$
\left|\frac{c_{\mu}}{\sqrt{c}}\right| \leq\left|\frac{\left(c^{2}\right)_{\tau} h_{x}}{2 c^{2} \sqrt{c}}\right|+\left|\frac{\left(c^{2}\right)_{x}}{2 c \sqrt{c}}\right| \leq O(1) \tau^{-\frac{\gamma+1}{4}},
$$

as above. Finally, estimating as above, we obtain

$$
\sqrt{c}\left(\frac{\widetilde{p}_{\mu}}{c}\right)_{h \mu}=O(1) \tau^{-\frac{\gamma+1}{4}}
$$

as required.

Since all terms in (3.13) are uniformly bounded, it follows that we can choose $\widehat{N}$ so that $(3.12)$ holds. Moreover, $\widehat{N}$ is independent of $N_{\rho L}$. The remainder of the proof follows that of Theorem 2.4 . 


\section{Compressible flow in a variable area duct}

\subsection{Equations and coordinates}

In this section, we first consider inviscid compressible flow in a duct of varying cross section $a\left(x^{\prime}\right)$. In Eulerian (spatial) coordinates $\left(x^{\prime}, t^{\prime}\right)$, this flow is described by

$$
\begin{aligned}
a_{t^{\prime}} & =0 \\
(a \rho)_{t^{\prime}}+(a \rho u)_{x^{\prime}} & =0 \\
(a \rho u)_{t^{\prime}}+\left(a \rho u^{2}\right)_{x^{\prime}}+a p_{x^{\prime}} & =0 \\
(a \rho E)_{t^{\prime}}+(a \rho E u+a p u)_{x^{\prime}} & =0
\end{aligned}
$$

$a=a\left(x^{\prime}\right)$ is the cross-sectional area of the duct and $E=e+\frac{1}{2} u^{2}$ is the total energy [6, 7, 12]. The others are the standard thermodynamic variables, subject to (2.4) as usual. The first equation describes the fixed duct, while the others represent conservation of mass, momentum and energy, respectively. For $C^{1}$ solutions, the energy equation is equivalent to an entropy equation,

$$
S_{t^{\prime}}+u S_{x^{\prime}}=0
$$

We use Lagrangian (material) coordinates $(x, t)$, defined by

$$
x=\int a\left(x^{\prime}\right) \rho\left(x^{\prime}\right) d x^{\prime}, \quad t=t^{\prime}
$$

so that

$$
d x=a \rho d x^{\prime}-a \rho u d t^{\prime}, \text { and } \quad d t=d t^{\prime} .
$$

We define a specific length by

$$
v:=\frac{1}{a \rho}
$$

and rewrite system (4.1) in the Lagrangian frame, to get

$$
\begin{aligned}
v_{t}-u_{x} & =0 \\
u_{t}+a p_{x} & =0 \\
S_{t} & =0
\end{aligned}
$$

valid for $C^{1}$ solutions, which is a variant of the generalized $p$-system (3.1).

We restrict our attention to a polytropic ideal gas (2.6), so that

$$
p=p(v, a, S)=K e^{\frac{S}{c_{\tau}}} a^{-\gamma} v^{-\gamma},
$$


and define the generalized Lagrangian wavespeed by

$$
C=\sqrt{-a p_{v}}=\sqrt{K \gamma} a^{-\frac{\gamma-1}{2}} v^{-\frac{\gamma+1}{2}} e^{\frac{S}{2 c_{\tau}}} .
$$

As above, we define new variables $m$ and $z$ for $S$ and $v$ by

$$
m=e^{\frac{S}{2 c_{\tau}}} \quad \text { and } \quad z=\int_{v}^{\infty} \frac{C a^{\frac{\gamma-1}{2}}}{m} d v=\frac{2 \sqrt{K \gamma}}{\gamma-1} v^{-\frac{\gamma-1}{2}} .
$$

It follows that

$$
\begin{aligned}
& v=K_{\tau} z^{-\frac{2}{\gamma-1}}, \quad p=K_{p} a^{-\gamma} m^{2} z^{\frac{2 \gamma}{\gamma-1}} \\
& \text { and } \quad C=C(m, z, a)=K_{c} a^{-\frac{\gamma-1}{2}} m z^{\frac{\gamma+1}{\gamma-1}}
\end{aligned}
$$

where the constants $K$ are given by (2.11). In these coordinates, $C^{1}$ solutions of (4.4) satisfy the equivalent system

$$
\begin{aligned}
z_{t}+\frac{C}{m} a^{\frac{\gamma-1}{2}} u_{x} & =0, \\
u_{t}+m C a^{-\frac{\gamma-1}{2}} z_{x}+2 \frac{a p}{m} m_{x}-\gamma p a_{x} & =0, \\
m_{t} & =0 .
\end{aligned}
$$

\subsection{Coupled Riccati equations}

Following [2], we define gradient variables by

$$
\begin{aligned}
& \alpha=u_{x}+a^{-\frac{\gamma-1}{2}} m z_{x}+\frac{\gamma-1}{\gamma} a^{-\frac{\gamma-1}{2}} m_{x} z, \\
& \beta=u_{x}-a^{-\frac{\gamma-1}{2}} m z_{x}-\frac{\gamma-1}{\gamma} a^{-\frac{\gamma-1}{2}} m_{x} z,
\end{aligned}
$$

and denote derivatives along forward and backward characteristics by

$$
\partial_{+}=\partial_{t}+C \partial_{x} \quad \text { and } \quad \partial_{-}=\partial_{t}-C \partial_{x}
$$

respectively.

Although the area $a\left(x^{\prime}\right)$ of the duct is stationary in spatial coordinates, it is moving in material coordinates. We write

$$
\dot{a}:=\frac{d a\left(x^{\prime}\right)}{d x^{\prime}} \quad \text { and } \quad \ddot{a}:=\frac{d^{2} a\left(x^{\prime}\right)}{d x^{\prime 2}},
$$

so that, according to (4.2), $a(x, t)$ satisfies

$$
a_{t}=u(x, t) \dot{a}\left(x^{\prime}\right), \quad a_{x}=v(x, t) \dot{a}\left(x^{\prime}\right) \quad \text { and } \quad a_{x x}=v^{2} \ddot{a}+v_{x} \dot{a},
$$

for $x^{\prime}=x^{\prime}(x, t)$. 
Lemma 4.1. For $C^{1}$ solutions of (4.1), we have

$$
\begin{aligned}
& \partial_{+} \alpha=k_{1}\left(k_{2}(3 \alpha+\beta)+\left(\alpha \beta-\alpha^{2}\right)\right)+k_{3}^{+}(\alpha-\beta)+F(x, t), \\
& \partial_{-} \beta=k_{1}\left(-k_{2}(\alpha+3 \beta)+\left(\alpha \beta-\beta^{2}\right)\right)+k_{3}^{-}(\beta-\alpha)+F(x, t),
\end{aligned}
$$

where

$$
\begin{gathered}
k_{1}=\frac{\gamma+1}{2(\gamma-1)} K_{c} z^{\frac{2}{\gamma-1}}, \quad k_{2}=\frac{\gamma-1}{\gamma(\gamma+1)} m_{x} z a^{-\frac{\gamma-1}{2}}, \\
k_{3}^{ \pm}=-\frac{\gamma-1}{4} u a^{-1} \dot{a} \pm \frac{3(\gamma-1)^{2}}{8} m z a^{-\frac{\gamma+1}{2}} \dot{a}
\end{gathered}
$$

and where

$$
F(x, t)=\frac{(\gamma-1)^{3}}{8 K_{c}} m^{2} z^{\frac{2 \gamma-4}{\gamma-1}} a^{-\gamma-1}\left(a \ddot{a}-\gamma \dot{a}^{2}\right)+\frac{(\gamma-1)^{2}}{2 \gamma} m m_{x} z^{2} a^{-\gamma} \dot{a} .
$$

We note that the coefficients $k_{j}$ and zero-order terms $F$ depend only on the solution itself and on stationary derivatives of initial entropy and duct shape. It is reasonable to assume that $|\dot{a}|,|\ddot{a}|$ and $\left|m_{x}\right|$ are uniformly bounded, so the coefficients and inhomogeneity are essentially zero order terms. As in all of the simpler examples, the quadratic nonlinearity drives the growth of $\alpha$ and/or $\beta$ to infinity, at which time derivatives blow up and shocks form in the solution.

Proof. We derive the equation for $\alpha$. Differentiating (4.7), we get

$$
\begin{aligned}
\partial_{+} \alpha=\left[u_{x t}\right. & \left.+C\left(a^{-\frac{\gamma-1}{2}} m z_{x}\right)_{x}\right]+\left[\left(a^{-\frac{\gamma-1}{2}} m z_{x}\right)_{t}+C u_{x x}\right] \\
& +\frac{\gamma-1}{\gamma}\left[\left(a^{-\frac{\gamma-1}{2}} m_{x} z\right)_{t}+C\left(a^{-\frac{\gamma-1}{2}} m_{x} z\right)_{x}\right] .
\end{aligned}
$$

Now differentiate (4.6) in $x$ to get

$$
\begin{aligned}
& \left(a^{-\frac{\gamma-1}{2}} m z_{x}\right)_{t}+C u_{x x}=-C_{x} u_{x}-\left(a^{-\frac{\gamma-1}{2}} m\right)_{x} z_{t}+\left(a^{-\frac{\gamma-1}{2}}\right)_{t} m z_{x}, \\
& u_{x t}+C\left(m a^{-\frac{\gamma-1}{2}} z_{x}\right)_{x}=-\left(2 \frac{a p}{m} m_{x}\right)_{x}+\left(\gamma p a_{x}\right)_{x}-C_{x} m a^{-\frac{\gamma-1}{2}} z_{x},
\end{aligned}
$$

and substitute in to get

$$
\begin{aligned}
\partial_{+} \alpha=- & \left(2 \frac{a p}{m} m_{x}\right)_{x}+\left(\gamma p a_{x}\right)_{x}-C_{x} m a^{-\frac{\gamma-1}{2}} z_{x} \\
& -C_{x} u_{x}-\left(a^{-\frac{\gamma-1}{2}} m\right)_{x} z_{t}+\left(a^{-\frac{\gamma-1}{2}}\right)_{t} m z_{x} \\
& +\frac{\gamma-1}{\gamma}\left[\left(a^{-\frac{\gamma-1}{2}} m_{x} z\right)_{t}+C\left(a^{-\frac{\gamma-1}{2}} m_{x} z\right)_{x}\right] .
\end{aligned}
$$

Note that we have eliminated all second derivatives except for $a_{x x}$ and $m_{x x}$, since $m_{x t}=0$, and we use (4.8) to eliminate $a_{t}, a_{x}$ and $a_{x x}$. The quadratic terms in (4.9) are those involving $C_{x}$. 
Finally, we express the derivatives $u_{x}, z_{t}$ and $z_{x}$ in terms of $\alpha$ and $\beta$. By (4.7), (4.6) and (4.5), we have

$$
\begin{aligned}
& u_{x}=\frac{1}{2}(\alpha+\beta), \\
& z_{x}=\frac{a^{\frac{\gamma-1}{2}}}{2 m}(\alpha-\beta)-\frac{\gamma-1}{\gamma m} z m_{x}, \\
& z_{t}=-\frac{K_{c}}{2} z^{\frac{\gamma+1}{\gamma-1}}(\alpha+\beta) .
\end{aligned}
$$

Substituting these into (4.10) and simplifying yields (4.9). We omit the details, which are similar to those appearing in [2].

\subsection{Decoupled Riccati equations}

As in [2, 4], equations (4.9) can be rewritten as a decoupled system with varying coefficients. Since the $\alpha$ equation has terms linear in $\beta$, these can be eliminated by an integrating factor. By (4.5), (4.6) and (4.7),

$$
\partial_{+} z=z_{t}+C z_{x}=-K_{c} z^{\frac{\gamma+1}{\gamma-1}}\left(\beta+\frac{\gamma-1}{\gamma} m_{x} z a^{-\frac{\gamma-1}{2}}\right),
$$

so that

$$
\beta=-\frac{1}{K_{c}} z^{-\frac{\gamma+1}{\gamma-1}} \partial_{+} z-\frac{\gamma-1}{\gamma} m_{x} z a^{-\frac{\gamma-1}{2}} .
$$

We substitute for $\beta$ in (4.9), move terms including $\partial_{+} z$ to the left hand side, and multiply by $z^{\frac{\gamma+1}{2(\gamma-1)}}$, to get

$$
\begin{aligned}
& \partial_{+}\left(z^{\frac{\gamma+1}{2(\gamma-1)}} \alpha\right)+\frac{1}{2 \gamma} m_{x} a^{-\frac{\gamma-1}{2}} z^{\frac{\gamma+1}{2(\gamma-1)}} \partial_{+} z-\frac{k_{3}^{+}}{K_{c}} z^{-\frac{\gamma+1}{2(\gamma-1)}} \partial_{+} z \\
&= k_{1}\left(k_{2}\left(3 \alpha-\frac{\gamma-1}{\gamma} m_{x} z a^{-\frac{\gamma-1}{2}}\right)+\left(-\frac{\gamma-1}{\gamma} m_{x} z a^{-\frac{\gamma-1}{2}} \alpha-\alpha^{2}\right)\right) z^{\frac{\gamma+1}{2(\gamma-1)}} \\
& \quad+k_{3}^{+}\left(\alpha+\frac{\gamma-1}{\gamma} m_{x} z a^{-\frac{\gamma-1}{2}}\right) z^{\frac{\gamma+1}{2(\gamma-1)}}+F(x, t) z^{\frac{\gamma+1}{2(\gamma-1)}}
\end{aligned}
$$

Lemma 4.2. There are variables $Y$ and $Q$, defined by

$$
\begin{aligned}
& Y=z^{\frac{\gamma+1}{2(\gamma-1)}} \alpha+\widetilde{Y}\left(z, u, m, m_{x}, a, \dot{a}\right), \\
& Q=z^{\frac{\gamma+1}{2(\gamma-1)}} \beta+\widetilde{Q}\left(z, u, m, m_{x}, a, \dot{a}\right),
\end{aligned}
$$

which satisfy the equations

$$
\begin{aligned}
& \partial_{+} Y=d_{0}+d_{1} Y+d_{2} Y^{2}, \\
& \partial_{-} Q=\bar{d}_{0}-\bar{d}_{1} Q+d_{2} Q^{2},
\end{aligned}
$$


with coefficients

$$
\begin{aligned}
& d_{2}=-\frac{\gamma+1}{\gamma-1} K_{c} z^{\frac{3-\gamma}{2(\gamma-1)}}, \\
& d_{1}, \bar{d}_{1}=d_{1}, \bar{d}_{1}\left(z, u, m, m_{x}, a, \dot{a}\right), \quad \text { and } \\
& d_{0}, \bar{d}_{0}=d_{0}, \bar{d}_{0}\left(z, u, m, m_{x}, m_{x x}, a, \dot{a}, \ddot{a}\right) .
\end{aligned}
$$

Proof. For convenience, we take $\gamma \neq 5 / 3$ or 3 . Writing (4.11) as a derivative along the characteristic, we get

$$
\begin{aligned}
& \partial_{+}\left(z^{\frac{\gamma+1}{2(\gamma-1)}} \alpha\right)+\partial_{+}\left(\frac{\gamma-1}{\gamma(3 \gamma-1)} m_{x} a^{-\frac{\gamma-1}{2}} z^{\frac{3 \gamma-1}{2(\gamma-1)}}\right) \\
& \quad-\frac{3(\gamma-1)^{2}}{8 K_{c}} m \dot{a} a^{-\frac{\gamma+1}{2}} z^{\frac{\gamma-3}{2(\gamma-1)}} \partial_{+} z+\partial_{+}\left(\frac{(\gamma-1)^{2}}{2 K_{c}(\gamma-3)} u a^{-1} \dot{a} z^{\frac{\gamma-3}{2(\gamma-1)}}\right) \\
& =\frac{(\gamma-1)^{2}}{2 K_{c}(\gamma-3)} a^{-1} \dot{a} z^{\frac{\gamma-3}{2(\gamma-1)}} \partial_{+} u \\
& \quad+\partial_{+}\left(\frac{\gamma-1}{\gamma(3 \gamma-1)} m_{x} a^{-\frac{\gamma-1}{2}}\right) z^{\frac{3 \gamma-1}{2(\gamma-1)}}+\frac{(\gamma-1)^{2}}{2 K_{c}(\gamma-3)} u z^{\frac{\gamma-3}{2(\gamma-1)}} \partial_{+}\left(a^{-1} \dot{a}\right) \\
& \quad+k_{1}\left(k_{2}\left(3 \alpha-\frac{\gamma-1}{\gamma} m_{x} z a^{-\frac{\gamma-1}{2}}\right)+\left(-\frac{\gamma-1}{\gamma} m_{x} z a^{-\frac{\gamma-1}{2}} \alpha-\alpha^{2}\right)\right) z^{\frac{\gamma+1}{2(\gamma-1)}} \\
& \quad+k_{3}^{+}\left(\alpha+\frac{\gamma-1}{\gamma} m_{x} z a^{-\frac{\gamma-1}{2}}\right) z^{\frac{\gamma+1}{2(\gamma-1)}}+F(x, t) z^{\frac{\gamma+1}{2(\gamma-1)}}
\end{aligned}
$$

Next, we eliminate the $\partial_{+} u$ term, which comes from differentiating $k_{3}^{+}$. By (4.6), we have

$$
\partial_{+} u=u_{t}+C u_{x}=-m a^{-\frac{\gamma-1}{2}} \partial_{+} z-\frac{\gamma-1}{\gamma} C z m_{x} a^{-\frac{\gamma-1}{2}}+\gamma p a_{x},
$$

and we again write this as a total derivative plus zero-order terms in $z$ and $u$.

Finally, we define

$$
\begin{aligned}
Y= & z^{\frac{\gamma+1}{2(\gamma-1)}} \alpha+\frac{(\gamma-1)^{2}}{2 K_{c}(\gamma-3)} u a^{-1} \dot{a} z^{\frac{\gamma-3}{2(\gamma-1)}}+\frac{\gamma-1}{\gamma(3 \gamma-1)} m_{x} a^{-\frac{\gamma-1}{2}} z^{\frac{3 \gamma-1}{2(\gamma-1)}} \\
& -\frac{(3 \gamma-13)(\gamma-1)^{3}}{4 K_{c}(\gamma-3)(3 \gamma-5)} m \dot{a} a^{-\frac{\gamma+1}{2}} z^{\frac{3 \gamma-5}{2(\gamma-1)}}
\end{aligned}
$$


and simplify, to get

$$
\begin{aligned}
\partial_{+} Y & =-z^{\frac{3 \gamma-5}{2(\gamma-1)}} \partial_{+}\left(\frac{(3 \gamma-13)(\gamma-1)^{3}}{4 K_{c}(\gamma-3)(3 \gamma-5)} m \dot{a} a^{-\frac{\gamma+1}{2}}\right) \\
& +\frac{(\gamma-1)^{2}}{2 K_{c}(\gamma-3)} a^{-1} \dot{a} z^{\frac{\gamma-3}{2(\gamma-1)}}\left(-\frac{\gamma-1}{\gamma} C z m_{x} a^{-\frac{\gamma-1}{2}}+\gamma p a_{x}\right) \\
& +\left(\frac{\gamma-1}{\gamma(3 \gamma-1)} m_{x} a^{-\frac{\gamma-1}{2}}\right) z^{\frac{3 \gamma-1}{2(\gamma-1)}}+\frac{(\gamma-1)^{2}}{2 K_{c}(\gamma-3)} u z^{\frac{\gamma-3}{2(\gamma-1)}} \partial_{+}\left(a^{-1} \dot{a}\right) \\
& +k_{1}\left(k_{2}\left(3 \alpha-\frac{\gamma-1}{\gamma} m_{x} z a^{-\frac{\gamma-1}{2}}\right)+\left(-\frac{\gamma-1}{\gamma} m_{x} z a^{-\frac{\gamma-1}{2}} \alpha-\alpha^{2}\right)\right) z^{\frac{\gamma+1}{2(\gamma-1)}} \\
& +k_{3}^{+}\left(\alpha+\frac{\gamma-1}{\gamma} m_{x} z a^{-\frac{\gamma-1}{2}}\right) z^{\frac{\gamma+1}{2(\gamma-1)}}+F(x, t) z^{\frac{\gamma+1}{2(\gamma-1)}} .
\end{aligned}
$$

Using (4.13) to write

$$
Y=z^{\frac{\gamma+1}{2(\gamma-1)}} \alpha+y_{0}, \quad \text { so } \quad \alpha=z^{-\frac{\gamma+1}{2(\gamma-1)}} Y-z^{-\frac{\gamma+1}{2(\gamma-1)}} y_{0},
$$

we eliminate $\alpha$ to get

$$
\partial_{+} Y=d_{0}+d_{1} Y+d_{2} Y^{2}
$$

with coefficients $d_{i}$ containing no derivatives of $z, u$, and in particular,

$$
d_{2}=-\frac{\gamma+1}{\gamma-1} K_{c} z^{\frac{3-\gamma}{2(\gamma-1)}} .
$$

Using $z^{\frac{2}{\gamma-1}}=K_{\tau} a \rho$, after a tedious calculation we get, for $\gamma \neq 5 / 3$ or 3 ,

$$
\begin{aligned}
d_{0}= & a^{\frac{\gamma-3}{4}}\left(\frac{1}{\gamma} K_{c} K_{\tau}^{\frac{5 \gamma+1}{4}}\left(\frac{\gamma-1}{3 \gamma-1} m m_{x x}-\frac{(3 \gamma+1)(\gamma-1)}{(3 \gamma-1)^{2}} m_{x}^{2}\right) a^{2} \rho^{\frac{5 \gamma+1}{4}}\right. \\
& +L_{1} m_{x} \dot{a} u \rho^{\frac{3 \gamma-1}{4}}+L_{2} m m_{x} \dot{a} \rho^{\frac{5 \gamma-3}{4}} \\
& +L_{3} m \dot{a}^{2} a^{-2} u \rho^{\frac{3 \gamma-5}{4}}+L_{4} m \ddot{a} a^{-1} u \rho^{\frac{3 \gamma-5}{4}} \\
& +L_{5} m^{2} a^{-2} \dot{a}^{2} \rho^{\frac{5 \gamma-7}{4}}+L_{6} \ddot{a} a^{-1} u^{2} \rho^{\frac{\gamma-3}{4}} \\
& \left.+L_{7} a^{-2} \dot{a}^{2} u^{2} \rho^{\frac{\gamma-3}{4}}+L_{8} m^{2} \ddot{a} a^{-1} \rho^{\frac{5 \gamma-7}{4}}\right), \\
d_{1}= & L_{9} m_{x} a \rho^{\frac{\gamma+1}{2}}+L_{10} u a^{-1} \dot{a}+L_{11} m a^{-1} \dot{a} \rho^{\frac{\gamma-1}{2}}, \\
d_{2}= & -\frac{\gamma+1}{\gamma-1} K_{c} K_{\tau}^{\frac{3-\gamma}{4}} a^{\frac{3-\gamma}{4}} \rho^{\frac{3-\gamma}{4}}<0,
\end{aligned}
$$

where the $L_{j}$ are constants depending only on $\gamma$.

Similarly, if $Q$ is defined by

$$
\begin{aligned}
Q= & z^{\frac{\gamma+1}{2(\gamma-1)}} \alpha+\frac{(\gamma-1)^{2}}{2 K_{c}(\gamma-3)} u a^{-1} \dot{a} z^{\frac{\gamma-3}{2(\gamma-1)}}-\frac{\gamma-1}{\gamma(3 \gamma-1)} m_{x} a^{-\frac{\gamma-1}{2}} z^{\frac{3 \gamma-1}{2(\gamma-1)}} \\
& +\frac{(3 \gamma-13)(\gamma-1)^{3}}{4 K_{c}(\gamma-3)(3 \gamma-5)} m \dot{a} a^{-\frac{\gamma+1}{2}} z^{\frac{3 \gamma-5}{2(\gamma-1)}}
\end{aligned}
$$


then it satisfies

$$
\partial_{-} Q=\bar{d}_{0}+\bar{d}_{1} Q+d_{2} Q^{2}
$$

with, for $\gamma \neq 5 / 3$ or 3 ,

$$
\begin{aligned}
\bar{d}_{0}= & a^{\frac{\gamma-3}{4}}\left(\frac{1}{\gamma} K_{c} K_{\tau}^{\frac{5 \gamma+1}{4}}\left(\frac{\gamma-1}{3 \gamma-1} m m_{x x}-\frac{(3 \gamma+1)(\gamma-1)}{(3 \gamma-1)^{2}} m_{x}^{2}\right) a^{2} \rho^{\frac{5 \gamma+1}{4}}\right. \\
& +\bar{L}_{1} m_{x} \dot{a} u \rho^{\frac{3 \gamma-1}{4}}+\bar{L}_{2} m m_{x} \dot{a} \rho^{\frac{5 \gamma-3}{4}} \\
& +\bar{L}_{3} m \dot{a}^{2} a^{-2} u \rho^{\frac{3 \gamma-5}{4}}+\bar{L}_{4} m \ddot{a} a^{-1} u \rho^{\frac{3 \gamma-5}{4}} \\
& +\bar{L}_{5} m^{2} a^{-2} \dot{a}^{2} \rho^{\frac{5 \gamma-7}{4}}+\bar{L}_{6} \ddot{a} a^{-1} u^{2} \rho^{\frac{\gamma-3}{4}} \\
& \left.+\bar{L}_{7} a^{-2} \dot{a}^{2} u^{2} \rho^{\frac{\gamma-3}{4}}+\bar{L}_{8} m^{2} \ddot{a} a^{-1} \rho^{\frac{5 \gamma-7}{4}}\right), \\
\bar{d}_{1}= & \bar{L}_{9} m_{x} a \rho^{\frac{\gamma+1}{2}}+\bar{L}_{10} u a^{-1} \dot{a}+\bar{L}_{11} m a^{-1} \dot{a} \rho^{\frac{\gamma-1}{2}},
\end{aligned}
$$

and $\bar{L}_{j}$ are constants depending only on $\gamma$. It is clear that similar equations hold for $\gamma=5 / 3$ or 3 .

\subsection{Singularity formation}

Having obtained Riccati equations (4.12) for derivative variables, we obtain a gradient blowup result as in Theorem 2.4. The result states that a strong enough compressive wave will eventually form a shock, provided the state variables remain finite. Theorem 2.1 indicates that state variables should indeed remain bounded for all times, although a vacuum can form in infinite time [26, 5].

Corollary 4.3. Assume there exist some positive constants $M_{i}$, such that the duct and initial data satisfy

$$
\begin{gathered}
M_{1}<a(x, 0)<M_{2}, \quad|\dot{a}(x, 0)|<M_{3}, \quad|\ddot{a}(x, 0)|<M_{4}, \\
M_{5}<m(x, 0)<M_{6}, \quad\left|m_{x}(x, 0)\right|<M_{7}, \quad\left|m_{x x}(x, 0)\right|<M_{8},
\end{gathered}
$$

while the solutions satisfy the a priori bounds

$$
|u(x, t)|<M_{9}, \quad \rho(x, t)<M_{10},
$$

and $\rho(x, t)>M_{11}$ if $1<\gamma \leq 3$, for $(x, t) \in \mathbb{R} \times \mathbb{R}^{+}$. Then there exists a positive constant $\bar{N}$, depending on $\gamma$ and $M_{i}$, such that, if

$$
Y<-\bar{N} \quad \text { or } \quad Q<-\bar{N}
$$

somewhere in the initial data, then a singularity forms in finite time. 
Proof. The roots of (4.12), if they exist, are given by the quadratic formula

$$
\frac{-d_{1} \pm \sqrt{d_{1}^{2}-4 d_{0} d_{2}}}{2 d_{2}} \text { or } \frac{\bar{d}_{1} \pm \sqrt{\bar{d}_{1}^{2}-4 \bar{d}_{0} d_{2}}}{2 d_{2}}
$$

which have bounds depending on the ratios $\left|d_{1}\right| /\left|d_{2}\right|,\left|d_{0}\right| /\left|d_{2}\right|,\left|\bar{d}_{1}\right| /\left|d_{2}\right|$ and $\left|\bar{d}_{0}\right| /\left|d_{2}\right|$. It follows that these roots have a lower bound $-\bar{N}$ depending on the $M_{i}$, and the proof follows in the same way as Theorem 2.4.

\subsection{Spherically symmetric Euler equations}

The equations for compressible flow in a duct include spherically symmetric three-dimensional Euler flow as a special case, on the exterior of a ball. Using $r=\left|x^{\prime}\right|$ as the radial coordinate, the Euler equations reduce to the duct equations with $x$ replaced by $r$ and $a(r)=r^{2}$.

In order to get cleaner equations, we consider the isentropic case, with $m=1$, say, and $\gamma \neq 5 / 3$ or 3 . With these assumptions, $m_{x}=0, \dot{a}=2 r$ and $\ddot{a}=2$, and (4.12) hold with coefficients

$$
\begin{aligned}
& d_{0}=r^{\frac{\gamma-3}{2}-2} \rho^{\frac{\gamma-3}{4}}\left(G_{1} u \rho^{\frac{\gamma-1}{2}}+G_{2} \rho^{\gamma-1}+G_{3} u^{2}\right) \\
& d_{1}=r^{-1}\left(G_{4} u+G_{5} \rho^{\frac{\gamma-1}{2}}\right) \\
& d_{2}=-\frac{\gamma+1}{\gamma-1} K_{c} K_{\tau}^{\frac{3-\gamma}{4}} r^{\frac{3-\gamma}{2}} \rho^{\frac{3-\gamma}{4}},
\end{aligned}
$$

for constants $G_{i}$. Thus the ratios are

$$
\begin{aligned}
& \frac{d_{1}}{d_{2}}=r^{\frac{\gamma-5}{2}} \rho^{\frac{\gamma-3}{4}}\left(\hat{G}_{4} u+\hat{G}_{5} \rho^{\frac{\gamma-1}{2}}\right) \\
& \frac{d_{0}}{d_{2}}=r^{\gamma-5} \rho^{\frac{\gamma-3}{2}}\left(\hat{G}_{1} u \rho^{\frac{\gamma-1}{2}}+\hat{G}_{2} \rho^{\gamma-1}+\hat{G}_{3} u^{2}\right),
\end{aligned}
$$

for constants $\hat{G}_{i}$. It follows that the roots $\beta_{ \pm}=\frac{-d_{1} \pm \sqrt{d_{1}^{2}-4 d_{0} d_{2}}}{2 d_{2}}$ of (4.12) satisfy the bound

$$
\beta_{ \pm} \geq-r^{\frac{\gamma-5}{2}} \rho^{\frac{\gamma-3}{4}}\left(\hat{G}_{6}|u|+\hat{G}_{7} \rho^{\frac{\gamma-1}{2}}\right),
$$

for constants $\hat{G}_{j}$ depending only on the equation of state.

A gradient blowup result similar to Theorem 2.4 now follows exactly as before for the exterior problem when $1<\gamma<5$. 


\section{References}

[1] A. Bressan, Hyperbolic systems of conservation laws: the 1-dimensional Cauchy problem, (Oxford Univ. Press, Oxford 2000).

[2] G. Chen, Formation of singularity and smooth wave propagation for the non-isentropic compressible Euler equations, J. Hyperbolic Diff. Eqns., 8:4 (2011), 671-690.

[3] G. Chen, Erik Endres and Helge Kristan Jenssen, Pairwise wave interactions in ideal polytropic gases, Arch. Rat. Mech. Anal. 204:3 (2012), 787-836.

[4] G. Chen and R. Young, Smooth solutions and singularity formation for the inhomogeneous nonlinear wave equation, J. Diff. Eqns. 252:3 (2012), 2580-2595.

[5] G. Chen and R. Young, Shock free solutions for the compressible Euler equations, Submitted.

[6] R. Courant and K. O. Friedrichs, Supersonic flow and shock waves, (Wiley-Interscience, New York, 1948).

[7] C. Dafermos, Hyperbolic conservation laws in continuum physics, Third edition, (Springer-Verlag, Heidelberg 2010).

[8] J. Glimm, Solutions in the large for nonlinear hyperbolic systems of equations, Comm. Pure Appl. Math., 18 (1965) 697-715.

[9] H.K. Jenssen, Blowup for systems of conservation laws, SIAM J. Math. Anal. 31 (2000), no. 4, 894-908.

[10] H.K. Jenssen and R.C. Young, Gradient driven and singular flux blowup of smooth solutions to hyperbolic systems of conservation laws, J. Hyp. Diff. Eq. 1 (2004), 627-641.

[11] F. John, Formation of singularities in one-dimensional nonlinear wave propagation, Comm. Pure Appl. Math., 27 (1974) 377-405.

[12] J. Hong and B. Temple, A bound on the total variation of the conserved quantities for solutions of a general resonant nonlinear balance law, SIAM J. Appl. Math. 64:3, 819-857.

[13] P. Lax, Development of singularities of solutions of nonlinear hyperbolic partial differential equations, J. Math. Physics, 5:5, (1964) 611-614. 
[14] P. Lax, Hyperbolic systems of conservation laws and the mathematical theory of shock waves, Conf. Board Math. Sci., 11, (SIAM, 1973).

[15] T.-T. Li, Global classical solutions for quasilinear hyperbolic systems, (Wiley, New york, 1994).

[16] T.-T. Li, Y. Zhou and D.-X. Kong, Global classical solutions for general quasilinear hyperbolic systems with decay initial data, Nonlinear Analysis, Theory, Methods \& Applications, 28:8, (1997) 1299-1332.

[17] T. Li and T. Qin, Physics and partial differential equations, Volume 1 (in Chinese), Higher Education press, Beijing, 1997.

[18] L. Lin, H. Liu and T. Yang, Existence of globally bounded continuous solutions for nonisentropic gas dynamics equations, J. Math. Anal. Appl., 209, (1997) 492-506.

[19] Longwei Lin and Seakweng Vong, A note on the existence and nonexistence of globally bounded classical solutions for nonisentropic gas dynamics, Acta Math. Scientia 26 (2006), 537-540.

[20] F. Liu, Global smooth resolvability for one-dimensional gas dynamics systems, Nonlinear Anal. 36, (1999) 25-34,

[21] T.-P. Liu, The development of singularities in the nonlinear waves for quasi-linear hyperbolic partial differential equations, Jour. Diff. Equations, 33, (1979) 92-111.

[22] M. A. Rammaha, Formation of singularities in compressible fluids in two-space dimensions, Proc. Amer. Math. Soc. 107:3, (1989) 705-714.

[23] T. Sideris, Formation of singularities in three-dimensional compressible fluids, Commun. Math. Phys., 101, (1985) 475-485.

[24] J. Smoller, Shock waves and reaction-diffusion equations, (SpringerVerlag, New York 1982).

[25] B. Temple and R. Young, A paradigm for time-periodic sound wave propagation in the compressible Euler equations, Methods Appl. Anal., 16:3, (2009) 341-364.

[26] Robin Young, The p-system II: The vacuum, Evolution Equations (Warsaw) (R. Picard, M. Reissig, and W. Zajaczkowski, eds.), Banach Center, 2001, pp. 237-252. 
[27] Robin Young, Blowup of solutions and boundary instabilities in nonlinear hyperbolic equations, Comm. Math. Sci. 2 (2003), 269-292.

[28] Robin Young, Nonuniqueness of BV solutions of quasilinear hyperbolic systems, Submitted,, 2010.

[29] Robin Young, Convergence of characteristics and shock formation, In preparation, 2011.

[30] Robin Young and Walter Szeliga, Blowup with small BV data in hyperbolic conservation laws, Arch. Rat. Mech. Anal. 179 (2006), 31-54. 